\title{
Study of the radioecological sensitivity of rice to radioactive contamination
}

\author{
B. Pey ${ }^{1}$, C. Mercat-Rommens ${ }^{1}$, A. Audebert ${ }^{2}$ and J.C. Mouret ${ }^{3}$ \\ ${ }^{1}$ IRSN, Laboratoire d'Études Radioécologiques en Milieux Continental et Marin, \\ CE Cadarache, 13115 Saint Paul-Lez-Durance, France \\ ${ }^{2}$ CIRAD, Avenue Agropolis, 34398 Montpellier Cedex 5, France \\ ${ }^{3}$ INRA, Agro Montpellier, UMR Innovation, 2 place Viala, 34060 Montpellier Cedex 1, France
}

\begin{abstract}
This study focused on the radioecology of rice and explored how the French specificities of rice cultivation can be taken into account in risk assessment. The objective of the study was to characterize the radioecological sensitivity of rice with respect to an accidental release of radioactivity. We want to know if a uniform and specific deposit would involve the same contamination on representative paddy fields of Camargue. To model the transfer of the radionuclides in the rice caryopsis following an accidental atmospheric pollution, we used the classical modelling for the cereals which considers first the interception by the foliage (modelled by an interception ratio) then the translocation to the grain (modelled by a translocation factor). The values of the parameters (interception ratio, translocation factor and yield) were regionalized with the agronomical software ORIZA2000, developed by the IRRI (International Rice Research Institute). We partly calibrated ORIZA2000 for a French rice variety: Ariete, thanks to physiological data provided for International Cooperation Centre in Agronomical Research for Development (CIRAD). ORIZA2000 proposes a daily follow-up of the leaf area index which can be correlated with the interception ratio. Five simulations with various climates, irrigation managements, and technical uses have been inputted on ORIZA2000. The data inputted were extracted from the database AGROSYST from the French Institute for Agronomical Research (INRA) and from the CIRAD. We established two scenarios of contamination. The first one consisted in a single contamination of water. In this case, there is almost no difference between simulations. However, in each simulation (except for organic farming) it is relevant to notice that the fourth depletion of water leads to a significant variation of contamination. The second contamination scenario consisted in a double contamination of water and of air. In that case, technical practices are responsible of the highest source of variability of the grain contamination. The variability of the contamination is mainly due to the variability of the yield which has in the radioecological modelling a dilution effect. The rice produced by organic farming is more sensitive than the rice produced by conventional farming because of low yields.
\end{abstract}

\section{INTRODUCTION}

The present study was carried out within the framework of the agricultural chapter of SENSIB, which is a project aimed at evaluating the sensitivity of agricultural productions to an accidental release of radioactivity [1]. In this study, we wish to assess whether a uniform and specific deposit would lead an identical contamination of the rice caryopsis over the whole of the rice plantations of France. The zone of study was restricted to the rice ecosystem of the Camargue because rice production in France is mainly localized in this area $(98.3 \%)$. There are three routes of contamination of rice. The first route consists of a direct contamination from the air via the leaves. The second route consists of an indirect contamination by water. Finally, the third route involves contamination of the soil by a radioactive deposit, but this route is not addressed in the present study. While the study covers the case of ${ }^{137} \mathrm{Cs}$ and ${ }^{90} \mathrm{Sr}$, the results presented here only concern ${ }^{137} \mathrm{Cs}$ and the process of dry deposition. 


\section{MATERIALS AND METHODS}

\subsection{Agronomic knowledge of rice cultivation in France}

In Metropolitan France, rice cultivation is intensive and of the "irrigated area" type, i.e. the presence and level of water is controlled by the rice grower (on a worldwide scale, $25 \%$ of rice plantations use this practice). The annual farming cycle is as follows: during winter, from October to March, the paddy field is not used. Then the soil is prepared for flooding which generally takes place towards the end of April. Sowing is then carried out in early May. During all the cultivation period (until September), the management of the irrigation is optimized according to the rice growth and to the cultivation practises. Indeed, the water level in the rice field is controlled and depends on multiple factors: 1 ) the presence or not of a period of drying out for rooting, 2) the number of fertilizations and weedkiller treatments, which sometimes impose temporary draining, 3) the phenological stage of the crop, which imposes a fixed water depth, 4) the type of irrigation: continuous (with overflow) or discontinuous. Before harvesting, the paddy field is finally drained. In Camargue, two types of rice cultivation coexisted: organic farming and conventional farming, the former distinguished by the exclusion of the use of synthetic fertilizers and pesticides.

\subsection{Radioecological modelling}

\subsubsection{Foliar transfer}

As regards the foliar transfer of radioactivity from the air to rice, we used the following equation resulting from the ASTRAL model to evaluate the transfer of radionuclides in the food chain and terrestrial environment following an accidental atmospheric emission [2]. The activity $\mathrm{Cv}_{f o l}$ at time $\mathrm{t}$ after deposition is determined by the initial deposit of radioactivity, and takes into account the climatic conditions at the time of deposition, the biological growth (dilution of the activity) and the radioactive decay during the interval from deposition to harvesting

$$
C_{v}=D_{r} \cdot\left(K_{r} \cdot F T d s+\left(1-K_{r}\right) \cdot F T d h\right) \cdot e^{-\lambda R \cdot\left(D_{a t R}-D_{a t D}\right)} .
$$

Where:

- $t$, time elapsed since formation of the deposit (d),

- DatD, date of deposit (d), DatR, date of harvest (d),

$-\lambda r$, radioactive decay constant $\left(\mathrm{d}^{-1}\right)$,

- Dr, total deposit of radionuclide $\mathrm{r}$ on the whole area of soil and plants $\left(\mathrm{Bq} \cdot \mathrm{m}^{-2}\right)$,

- $K r$, proportion of dry deposit in the total deposit (dimensionless),

- FTds, dry deposit transfer factor $\left(\mathrm{m}^{2} \cdot \mathrm{kg}^{-1}\right.$ fresh weight),

- FTdh, wet deposit transfer facto $\left(\mathrm{m}^{2} \mathrm{~kg}^{-1}\right.$ fresh weight).

In ASTRAL, the aggregate factor which globally expresses the foliar transfer of radionuclides, FTD, depends at the same time on the parameters of deposition, crop yield and translocation. The methodology of the SENSIB project consists of regionalizing these parameters, i.e. studying the influence of their spatial variability on contamination of the harvest.

The deposition ratio is a dimensionless quantity obtained by dividing the activity absorbed by the vegetation by the total activity deposited on a surface of $1 \mathrm{~m}^{2}$. The deposition ratio can be estimated by the Leaf Area Index (LAI). The German model ECOSYS-87 [3] proposes a formula that depends partly on the LAI, which can be used for computing this deposition ratio in dry weather conditions:

$$
R C s=\frac{L A I / L A / I_{\max }}{\left(L A I / L A / I_{\max }\right)+\left(\mu_{s o l} / \mu_{\max }\right)}
$$


Where:

- $L A I$, leaf-area index at the time of formation of the deposit $\left(\mathrm{m}^{2} \cdot \mathrm{m}^{-2}\right)$,

- $L A I_{\text {max }}$, maximum leaf-area index $\left(\mathrm{m}^{2} \cdot \mathrm{m}^{-2}\right)$,

- $\mu_{\text {sol }}$, dry deposition velocity to the soil $\left(\mathrm{m} \cdot \mathrm{s}^{-1}\right)$,

- $\mu_{\max }$, maximum deposition velocity onto the aerial biomass $\left(\mathrm{m} \cdot \mathrm{s}^{-1}\right)$.

To regionalize the deposition data, we applied the ORYZA2000 software developed by the IRRI (International Rice Research Institute), which simulates the growth and development of rice cultivation with a daily time step. The input parameters required to carry out these simulations comprise daily climatic data, data on cultivated varieties, soil characteristics and the crop management sequence.

For the climatic data, we used two meteorological stations of the Camargue (Méjanes and Adrien). The model ORYZA2000 had been built for rice varieties not cultivated in France. Therefore, it was necessary to calibrate the model for varieties of rice cultivated in the Camargue thanks to the agrophysiological data obtained from CIRAD experimentations. A partial calibration was carried out for the Ariete variety. The information concerning crops management comes from the AGROSYST database of the Innovation Mixed Research Unit of the INRA in Montpellier. These data sets collected, over several years and many rice farms, parameters concerning crop systems, the rice growth, weeds, fertilization, plant-health treatments and yield. These data were sorted in order to identify the main types of crop management sequence, while focusing on the Ariete variety.

Six simulations were carried out with ORIZA2000. Simulations 1 and 2 are identical and are only used to calibrate the results. Simulations 2 and 3 allow us to identify the influence of the climate. In simulation 4 , we study the influence of water depth on surfaces likely to pick up contamination. In simulations 5 and 6, we simulate a chemically-treated crop under stale-seedbed management as well as an organic crop, using different dates of wetting, crop management systems and nitrogenous inputs.

As regards translocation, we use the values of Choi et al. [4], while taking into account the variability produced by the climate and cultivation methods, which lead to disparate results in terms of the duration required to reach different phenological stages (flowering, in particular).

\subsubsection{Transfer by water}

As regards the transfer of radioactivity of water to rice, there are no equations or models that simulate contamination via the roots and/or submerged parts of the plant. Hence, we use the transfer factor FTa $\left(\mathrm{m}^{2} \cdot \mathrm{kg}^{-1}\right)$, which represents the ratio between the specific activity of the grain at harvest time $\left(\mathrm{Bq} \cdot \mathrm{kg}^{-1}\right)$ and the deposit of activity per unit area $\left(\mathrm{Bq} \cdot \mathrm{m}^{-2}\right)$. This factor depends on the phenological stage of the plant and thus takes account of the physiological change of activity and the development of the different parts of the plant with time. The values used result from a review of the literature data $[5-8]$.

\section{RESULTS AND DISCUSSION}

One of the outputs computed by ORYZA2000 corresponds to the surface-area of luminous interceptio $\mathrm{n}$ (LAI) and not the physical interception. The water sheet is likely to cover part of the plant and thus reduce the surface-area intercepted by atmospheric pollutants. Thus, the LAI decreases in proportion to the ratio of water depth to height of rice. In this way, Figures 1 and 2 show the real surface-area of interception of the radionuclides and the corresponding transfer factor in aggregate dry weather conditions (FTds).

Several remarks can be made. The dominant factor affecting the variation of LAI is the crop management sequence. The fourth drying out period and the draining vary the LAI significantly in simulations 2, 3 and 6. The water depth influences the LAI to a lesser extent. The witness FTDs reaches a maximum at $0.102 \mathrm{~m}^{2} / \mathrm{kg}$ fresh weight. From June 28, we observe a clear difference in contamination 


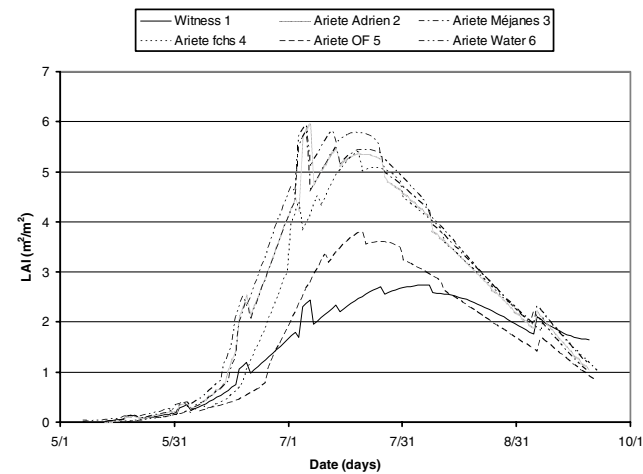

Figure 1. Variation of LAI with time Figure 2. Variation of FTds with time.

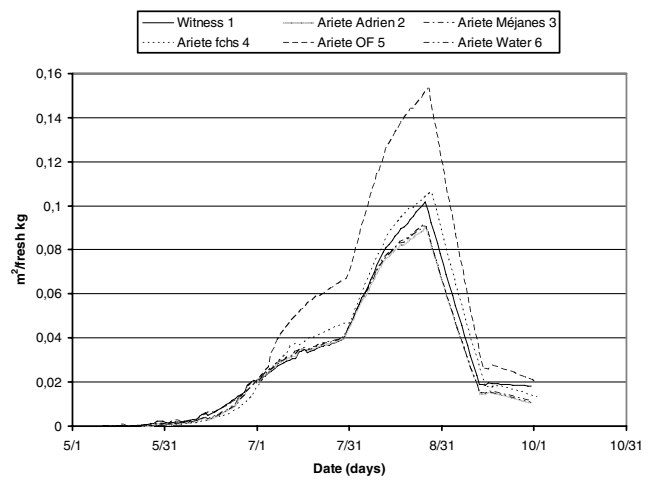

Figure 2. Variation of FTds with time.

according to the crop management sequence. Organic agriculture with low yields display the highest values of FTDs (approximately 1.7 times higher than for conventional agriculture). The yield acts here as a factor diluting the radioactive contamination. For organic productions, the yield is low and pollution is thus more concentrated in the grains than with the other simulations. The yield is therefore an important factor influencing radioecological sensitivity.

If we combine the two contamination routes (dual transfer by water and air towards rice), we obtain the curve given in Figure 3.

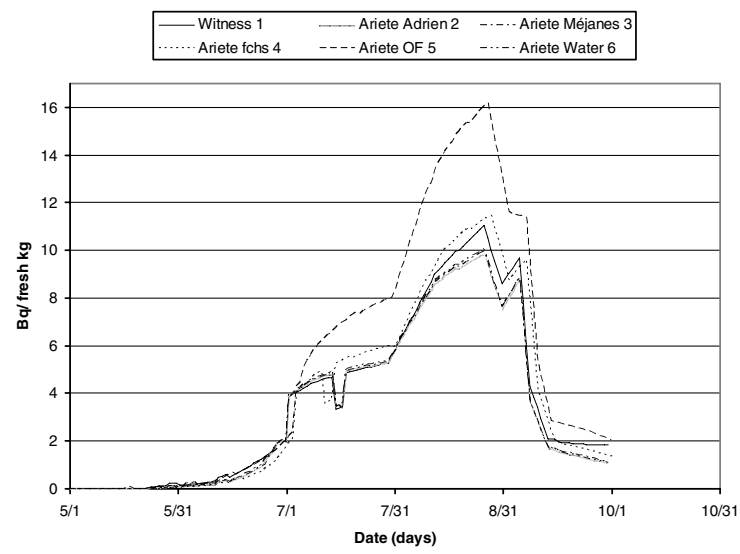

Figure 3. Contamination via dual route following dry deposition of $100 \mathrm{~Bq} \cdot \mathrm{m}^{-2}$ of ${ }^{137} \mathrm{Cs}$.

The contamination reaches $11 \mathrm{~Bq} \cdot \mathrm{kg}^{-1}$ for the control simulation. Apart from the fourth drying out period, which leads to a decrease in contamination for certain simulations, the curves have the same form as those obtained for foliar contamination. This shows the preponderance of foliar transfer compared to the transfer by water when the two routes coexist.

\section{CONCLUSION}

The aim of this study was to characterise and rank sensitivity factors of the Camargue rice growing ecosystem following an accidental radioactive pollution. To do this, it was necessary to select and 
calibrate a model of rice growth (ORYZA2000) to growing conditions in the Camargue. Accidental radioactive contamination can occur by three different pathways: air, soil and irrigation water. Contamination by the soil is negligible during the year of the contamination. The inclusion of regional specificities showed a period of heightened sensitivity at the time of rice seed maturation. Maximal leaf development associated with the mobilisation of resources to fill seeds explains why this period of the plant cycle results in higher contaminations. For an identical deposition, contamination by caesium predominated over that by strontium (the results from strontium are not illustrated in this paper). In the case of double pathway contamination (air and water), this study revealed contamination differences that depended on the conditions of deposition, i.e. dry or wet (the results for wet conditions are not illustrated in this paper). In the case of a dry deposition, leaf contamination by the air predominated. In this case and for caesium, the three sensitivity factors in order of importance are yield, a fourth draining (between June 29 and July 6 for the Ariete variety) and the culture calendar. Agricultural techniques play an important role in the levels of contamination. Organic farming productions are more highly contaminated than conventional cultures because of their lower yields. In addition, the shift in production cycles (maximum of 3 days for Ariete) is a source of variability that affects final contamination.

\section{References}

[1] Mercat-Rommens C., Roussel-Debet S., Briand B., Durand V., Besson B., Renaud P., La sensibilité radioécologique des territoires : vers un outil opérationnel - le projet SENSIB, Radioprotection 42 (2007) 277-295.

[2] Mourlon C., Calmon P., ASTRAL: a code for assessing situations after a nuclear accident, 12th annual meeting of SETAC Europe, challenges in environmental risk assessment and modelling: linking basic and applied research, Vienna (Austria), 12-16 may 2002.

[3] Müller H., Pröhl G., ECOSYS-87: a dynamic model for assessing radiological consequences of nuclear accidents, Health Physics 64 (1993) 232-252.

[4] Choi Y.H., Lim K.M., Yu D., Park H.G., Choi Y.G., Lee C.M., Transfer pathways of ${ }^{54} \mathrm{Mn},{ }^{57}$ Co, ${ }^{85} \mathrm{Sr},{ }^{103} \mathrm{Ru}$ and ${ }^{134} \mathrm{Cs}$ in rice and radish plants directly contaminated at different growth stages, Annals of Nuclear Energy 29 (2002) 429-446.

[5] Leung J.K.C., Shang Z.R., Uptake of ${ }^{137} \mathrm{Cs}$ and ${ }^{90} \mathrm{Sr}$ in rice plants, Health Physics 84 (2003) 170-179.

[6] Choi Y.H., Lim K.M., Choi H.J., Choi G.S., Lee H.S., Lee C.W., Plant uptake and downward migration of ${ }^{85} \mathrm{Sr}$ and ${ }^{137} \mathrm{Cs}$ after their deposition on to flooded rice fields: lysimeter experiments with and without the addition of $\mathrm{KCl}$ and lime, Journal of Environmental Radioactivity 78 (2005) 35-49.

[7] Choi Y.H., Lim K.M., Park H.G., Kang H.S., Lee H.S., Transfer of ${ }^{137}$ Cs to rice plants from various paddy soils contaminated under flooded conditions at different growth stages, Journal of Environmental Radioactivity 80 (2005) 45-58.

[8] Choi Y.H., Kang H.S., Jun I., Keum D.K., Park H.K., Choi G.S., Lee H., Lee C.W., Transfer of ${ }^{90} \mathrm{Sr}$ to rice plants after its acute deposition onto flooded paddy soils, Journal of Environmental Radioactivity 93 (2007) 157-169. 
